\title{
How do Singapore patients view post-anaesthesia adverse outcomes? A single-centre willingness-to-pay study
}

Xinqi $\underline{\text { Look }}^{1,2}$, MBBS, May Un Sam $\underline{\text { Mok }}^{2,3}$, MBChB, FRCA, Yan Sheng $\underline{\text { Tay }}^{4}$, Hairil Rizal Abdullah ${ }^{2,3}$, MBBS, MMed

INTRODUCTION Knowing how patients value the quality of anaesthesia helps anaesthesiologists to customise their service. However, generalising findings from Western population-based willingness-to-pay studies across different cultures and societies might result in the oversight of some contextualised perspectives of the anaesthesia experience. This study aimed to capture the Singapore perspective of undesired post-anaesthesia outcomes.

METHODS 132 patients recruited in a pre-anaesthetic evaluation clinic were given questionnaires describing ten possible post-anaesthetic outcomes. Outcomes were ranked for undesirability and assigned relative value through the hypothetical proportioning of SGD 100 to avoid their occurrence. Data was analysed with reference to patients' background and anaesthetic history.

RESULTS A response rate of $69.1 \%$ ( $n=132 / 191$ ) was achieved. Outcomes from the most to least undesirable were pain; vomiting; nausea; shivering; orodental trauma; sore throat; abrasions; somnolence; and thirst. Relative values allocated, in descending order, were pain; vomiting; nausea; orodental trauma; abrasions; sore throat; shivering; somnolence; and thirst. CONCLUSION Similar to previous studies in Western populations, pain, vomiting and nausea were the top three adverse outcomes that Singapore patients wished to avoid. However, discrepancies with Western patients were seen in spending attitudes, possibly accounted for by differences in healthcare socioeconomics. This study provided a better understanding of Singapore patients' perspectives on post-anaesthesia adverse outcomes and could help to improve treatment strategy and resource management.

Keywords: anaesthesia, attitude to health, post-anaesthesia adverse outcomes, willingness to pay

\section{INTRODUCTION}

Post-anaesthesia adverse outcomes, such as postoperative pain and nausea, are a common occurrence among many patients and have been associated with poor patient experience, longer hospitalisation, increased distress and higher healthcare costs. ${ }^{(1-5)}$ Research to increase understanding of patients' perspectives and expectations of care is needed to inform clinical practice guidelines. ${ }^{(6-10)}$ However, research has thus far largely been centred on the Western population, which limits the transferability of results to the Singapore population, as differences in culture, socioeconomics and healthcare structure have an undeniable influence on patients' perspectives, expectations of care and health-seeking behaviour. (11-16) Furthermore, there are significant differences between the willingness-to-pay spending habits of Asians and Westerners. It has been found that Asians are more willing to pay to avoid negative emotions, but less willing to do so to experience positive emotions, while Western populations have reported converse results. ${ }^{(17)}$

For this reason, examining the concerns and expectations of Singapore patients has practical significance for the tailoring of anaesthetic management. We aimed to investigate Singapore patients' perspectives on undesired post-anaesthesia outcomes and their willingness to pay to avoid these outcomes.

\section{METHODS}

This study was conducted at Singapore General Hospital (SGH), a 1,800-bed tertiary academic hospital in Singapore. Its methodology was reviewed by the SingHealth Centralised Institutional Review Board, which waived the need for written consent (CIRB/2014/822D).

The study was formulated in two languages, English and Mandarin, with back-translation by two bilingual experts for accuracy. Surveyed items comprised four sections: (a) demographics (e.g. age, gender, ethnicity, marital status, education, monthly household income, and private or subsidised); (b) anaesthesia history (e.g. previous anaesthesia, type of anaesthesia underwent previously, previous undesired outcomes experienced and current surgery planned); (c) ranking section; and (d) willingness to pay. No participant identifiers were recorded to ensure anonymity.

Based on a review of previous studies and samples of anaesthetic consent forms, a list of possible common postanaesthesia adverse outcomes had been formulated and used at our institution to counsel patients. We further reviewed this list, but chose to study only ten outcomes to improve the participation rate of this survey. Patients were asked to rank the ten listed postanaesthesia adverse outcomes in order of undesirability. Visual aids in the form of flashcards describing each outcome were

\footnotetext{
${ }^{1}$ Ministry of Health Holdings Pte Ltd, ${ }^{2}$ Department of Anaesthesiology, Singapore General Hospital, ${ }^{3}$ Duke-NUS Medical School, ${ }^{4}$ NUS Yong Loo Lin School of Medicine, National University of Singapore, Singapore

Correspondence: Dr Hairil Rizal Abdullah, Consultant, Department of Anaesthesiology, Singapore General Hospital, Outram Road, Singapore 169608. hairil.rizal.abdullah@singhealth.com.sg
} 
provided (Box 1). Descriptions were modified from previous study descriptors and from a pilot study. The following written and verbal instructions were given: "Below is a list of descriptions of what could occur after surgery in the recovery room. Assuming that each of the situations was equally likely to occur, please rank the following outcomes from 1 to 10, with 1 being what you would most want to avoid and 10 being what you would deem least important to avoid."

To determine their willingness to pay to avoid the outcomes, each patient was asked to hypothetically distribute SGD 100 across the outcomes, assigning greater value to more undesirable outcomes and exercising choice on which outcomes to spend on. The amount assigned to each particular outcome was used to determine their relative value. The following instruction was given in both written and verbal form: "You have $\$ 100$ to use to avoid the possible outcomes in the recovery room. The higher the amount you spend on an outcome, the lower the possibility that it will happen. Please distribute the $\$ 100$ according to your preference (with the smallest denomination being \$1), spending the money according to the outcomes you would most like to avoid. You do not need to spend on all items. You must spend all \$100 and nothing more."

Two trained interviewers administered the questionnaire in standardised interviews at the pre-anaesthetic evaluation clinic, over a three-week period in March 2015. Each interviewer was provided with a scripted document to administer the questionnaire and briefed on common queries to ensure consistency. The interviewers could approach the patients before or after the preoperative assessment consultation and anaesthesia consent process. The inclusion criteria were: (a) ability to give verbal consent; (b) above 21 years of age; and (c) ability to communicate or read in English and/or Mandarin. Those with cognitive disabilities that would affect comprehension were excluded.

Data was entered and statistically analysed using IBM SPSS Statistics version 20 (IBM Corp, Armonk, NY, USA). Frequency of demographics and clinical characteristics of patients were analysed as means and proportions. Outcome variables were evaluated using the modal score, median and interquartile ranges for ordinal rank, and the relative value of each item. Testing of internal validity and measurement of correlation was done using Spearman's rank correlation. Comparisons between subgroups were performed using paired $t$-test and Mann-Whitney $U$ test or Wilcoxon rank sum test. Statistical significance was set at a two-tailed $p<0.05$.

\section{RESULTS}

Clinic patients were sampled over a three-week period, resulting in a total of 191 patients, among whom 31 (16.2\%) declined participation, $11(5.8 \%)$ did not meet the inclusion criteria and $14(7.3 \%)$ had incomplete surveys. Among the 135 completed surveys, $3(2.2 \%)$ were discarded due to faulty information (i.e. patients ranked the 'normal' outcome higher than ten or the amount spent did not add up to SGD 100). 132 patients (response rate $69.1 \%$ ) were thus included in the subsequent analyses.

As shown in Table I, the mean age of our patients was $53 \pm 14$ (range 22-81) years. Patients were represented across the major ethnicities, both genders, various educational levels and income groups, and there were both subsidised and paying-class patients. The demographics of the study population were generally similar to that of surgical patients in SGH. In our study, $72.0 \%$ of the patients $(n=95)$ had a history of previous anaesthesia, of whom $85.3 \%$ had undergone general anaesthesia. Previous regional and local anaesthesia were reported by $22.1 \%$ and $9.5 \%$ of patients, respectively. Among patients with a history of previous anaesthetic use, $66.3 \%(n=63)$ recalled experiencing undesired outcomes, including nausea $(31.6 \%)$, pain $(30.5 \%)$ and vomiting (25.3\%). There was no recall of orodental trauma.

Fig. 1 shows the frequency distribution for the undesirability ranking of each outcome. Table II shows how our patients ranked the outcomes in descending order of undesirability and the

\footnotetext{
Box 1. List of post-anaesthesia adverse outcomes.

Nausea

Shivering

Vomiting

Somnolence

Sore throat

Pain

Abrasion

Orodental trauma

Thirst

Normal

When you wake up in the recovery room after surgery, you feel a strong desire to vomit, as if you are seasick. Any movement makes it worse.

When you wake up in the recovery room after surgery, you feel cold and your entire body is shaking uncontrollably. You are unable to hold a cup or speak properly from the shivering.

When you wake up in the recovery room after surgery, you are retching to the point that your abdominal muscles ache.

When you wake up in the recovery room after surgery, you are so sleepy that you are unable to stay awake long enough to tell the nurse how you are feeling.

When you wake up in the recovery room after surgery, your throat is painful, and it hurts to swallow or speak. When you wake up in the recovery room after surgery, you feel a constant sharp pain over your surgical wound. Any movement makes it worse.

When you wake up in the recovery room after surgery, you feel a stinging sensation over a patch of injured skin on your face (such as, cheek, eyelids or chin).

When you wake up in the recovery room after surgery, you are told that there was permanent damage done to your teeth, which will require dental review. You also notice a stinging cut on your lip.

When you wake up in the recovery room after surgery, you have a dry mouth and a strong desire to drink. However, you are not allowed to have any liquid.

When you wake up in the recovery room after surgery, you are alert, comfortable, without pain and aware of your surroundings.
} 
Table I. Demographic and clinical characteristics of patients $(n=132)$.

\begin{tabular}{|c|c|c|c|}
\hline Variable & No. (\%) & Variable & No. (\%) \\
\hline Age $(y r)^{*}$ & $53 \pm 14(22-81)$ & \multicolumn{2}{|l|}{ Patient classification } \\
\hline Gender & & Paying class & $41(31.1)$ \\
\hline Men & $64(48.5)$ & Subsidised & $91(68.9)$ \\
\hline Women & $68(51.5)$ & \multicolumn{2}{|l|}{ Anaesthetic history } \\
\hline Marital status & & Type of surgery planned & $132(100.0)$ \\
\hline Single & $23(17.4)$ & Urologic & $13(9.8)$ \\
\hline Married & 105 (79.5) & Cardiac & $0(0)$ \\
\hline Divorced/widowed & $4(3.0)$ & Neurosurgical & $2(1.5)$ \\
\hline Ethnicity & & Otolaryngological & $8(6.1)$ \\
\hline Chinese & $109(82.6)$ & General & $51(38.6)$ \\
\hline Malay & $11(8.3)$ & Orthopaedic & $49(37.1)$ \\
\hline Indian & $9(6.8)$ & Gynaecological & $4(3.0)$ \\
\hline Other & $3(2.3)$ & Other & $5(3.8)$ \\
\hline Highest education level & & History of previous anaesthesia & $95(72.0)$ \\
\hline None & $7(5.3)$ & \multicolumn{2}{|l|}{ Type of previous anaesthesia $(n=95)$} \\
\hline Primary education & $18(13.6)$ & General & $81(85.3)$ \\
\hline Secondary education & $46(34.8)$ & Regional & $21(22.1)$ \\
\hline Tertiary education: junior college/ & $25(18.9)$ & Local & $9(9.5)$ \\
\hline polytechnic/ITE & & \multirow{2}{*}{\multicolumn{2}{|c|}{$\begin{array}{l}\text { Previous undesired clinical outcome }{ }^{+} \\
(n=95)\end{array}$}} \\
\hline University degree & $26(19.7)$ & & \\
\hline Other & $10(7.6)$ & Nausea & $30(31.6)$ \\
\hline \multirow{2}{*}{$\begin{array}{l}\text { Monthly household } \\
\text { income (SGD) }\end{array}$} & & Shivering & $8(8.4)$ \\
\hline & & Vomiting & $24(25.3)$ \\
\hline Retired & $27(20.5)$ & Somnolence & $12(12.6)$ \\
\hline$<3,000$ & $21(15.9)$ & Sore throat & $8(8.4)$ \\
\hline $3,000-6,000$ & $37(28.0)$ & Pain & $29(30.5)$ \\
\hline $6,000-9,000$ & $20(15.2)$ & Abrasion & $3(3.2)$ \\
\hline $9,000-12,000$ & $6(4.5)$ & Orodental trauma & $0(0)$ \\
\hline$>12,000$ & $7(5.3)$ & Thirst & $18(18.9)$ \\
\hline Unspecified & $14(10.6)$ & None of the above & $32(33.7)$ \\
\hline
\end{tabular}

*Data presented as mean \pm standard deviation (range). tPatients may have more than one previous undesired clinical outcome. ITE: Institute of Technical Education

Table II. Ranking and relative value scores of post-anaesthesia adverse outcomes.

\begin{tabular}{|lllll|}
\hline Adverse outcome & Modal ranking & $\begin{array}{l}\text { Ranked as most undesired outcome } \\
{[\text { No. }(\%)](\mathbf{n = 1 3 2 )}}\end{array}$ & \multicolumn{2}{c|}{ Median (interquartile range) } \\
\cline { 4 - 5 } & & $63(47.7)$ & Ranking & Relative value (SGD) \\
\hline Pain & 1 (most undesirable) & $30(22.7)$ & $2.00(1.00-4.00)$ & $50(20-90)$ \\
\hline Vomiting & 2 & $13(9.8)$ & $3.00(2.00-4.75)$ & $20(10-40)$ \\
\hline Nausea & 3 & $7(5.3)$ & $6.00(4.00-7.00)$ & $20(10-50)$ \\
\hline Orodental trauma & 4 & $4(3.0)$ & $6.00(4.00-7.00)$ & $10(10-20)$ \\
\hline Shivering & 5 & $2(1.5)$ & $6.00(4.00-7.00)$ & $20(10-30)$ \\
\hline Abrasions & 6 & $2(1.5)$ & $6.00(4.00-7.00)$ & $11(10-25)$ \\
\hline Sore throat & 6 & $8(6.1)$ & $7.00(4.25-8.00)$ & $10(10-20)$ \\
\hline Somnolence & 8 & $3(2.3)$ & $8.00(6.00-9.00)$ & $10(10-20)$ \\
\hline Thirst & 9 & $0(0)$ & $10.00(10.00-10.00)$ & $0(0-0)$ \\
\hline Normal & 10 (least undesirable) & & \\
\hline
\end{tabular}

Data excludes surveyed participants who did not spend any amount on an outcome. Hence, calculations were based on those who paid to avoid the outcome.

relative value that reflected their willingness to pay to avoid each outcome. We found that $47.7 \%$ of patients $(n=63)$ ranked pain as their most adverse outcome to avoid, followed by vomiting ( $\mathrm{n}=30,22.7 \%$ ). To avoid pain (median amount SGD 50), our participants would spend two-thirds more when compared with vomiting (median amount SGD 30) and 2.5 times of the amount they would spend to avoid nausea (median amount SGD 20). Patients ranked shivering equal to orodental trauma, abrasions and sore throat at sixth place, yet were less willing to pay to avoid sore throat and shivering. The amount they were willing to pay to 

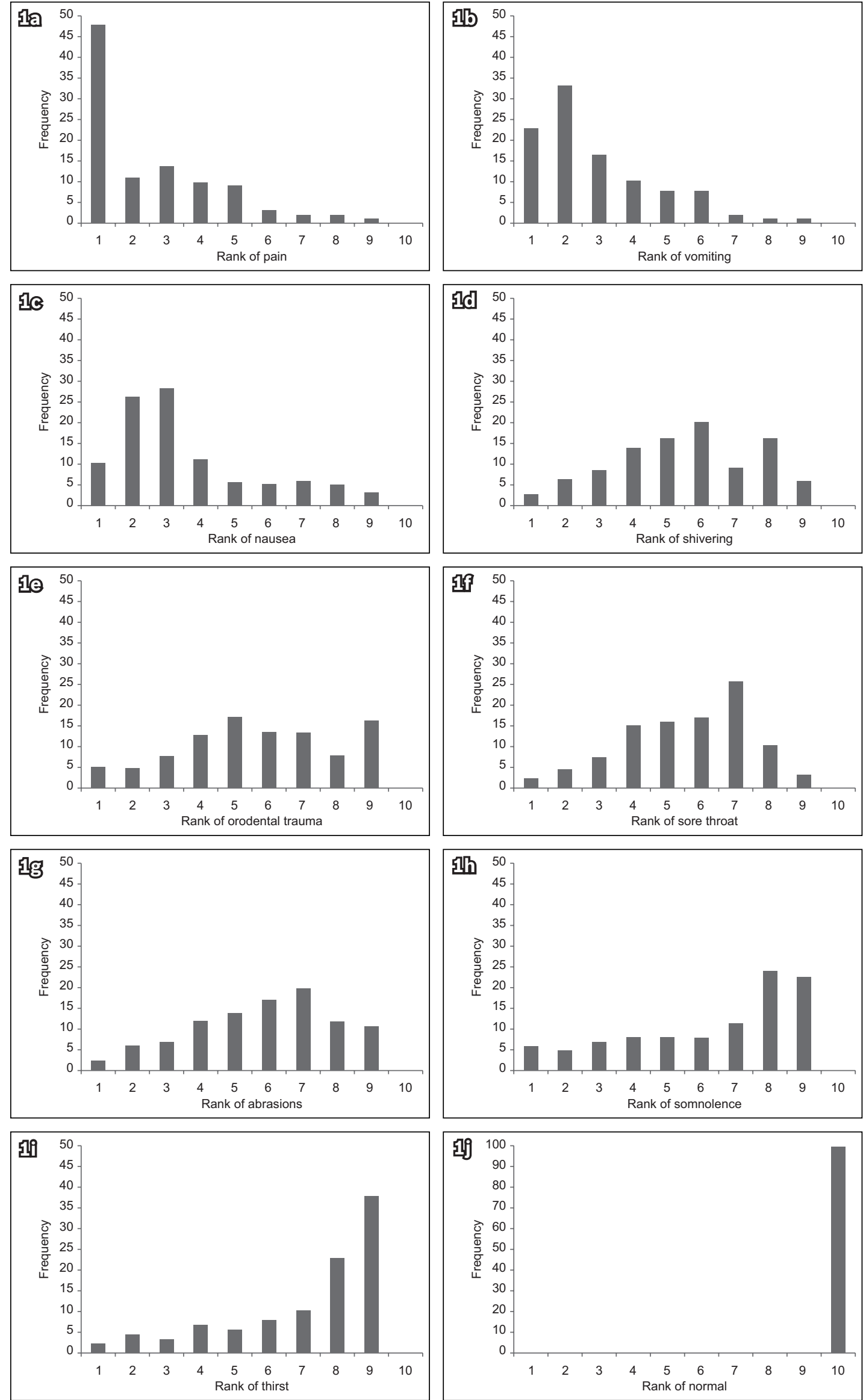

Fig. 1 Bar graphs show the frequency distribution for the undesirability ranking of each post-anaesthesia adverse outcome: (a) pain; (b) vomiting; (c) nausea; (d) shivering; (e) orodental trauma; (f) sore throat; (g) abrasion; (h) somnolence; (i) thirst; and (j) normal outcome. 
avoid orodental trauma and abrasions, SGD 20, was double the amount allocated to shivering (SGD 10) and sore throat (SGD 11). The normal outcome served as the reference point.

Using Spearman's rank correlation coefficient (r), we found associations between the ranks and the relative values of complications, such as pain $(r=-0.631, p<0.001)$, vomiting $(r=-0.487, p<0.001)$, nausea $(r=-0.601, p<0.001)$, orodental trauma $(r=-0.311, p=0.031)$ and somnolence $(r=-0.474$,

Table III. Correlation between outcome rank and relative value for adverse outcomes.

\begin{tabular}{|llll|}
\hline Adverse outcome & $\begin{array}{l}\text { No. of } \\
\text { patients }\end{array}$ & $\begin{array}{l}\text { Spearman's } \\
\text { rho }\end{array}$ & p-value \\
\hline Nausea & 61 & -0.601 & $<0.001$ \\
\hline Shivering & 25 & -0.309 & 0.133 \\
\hline Vomiting & 73 & -0.487 & $<0.001$ \\
\hline Somnolence & 21 & -0.474 & 0.030 \\
\hline Sore throat & 31 & -0.282 & 0.125 \\
\hline Pain & 95 & -0.631 & $<0.001$ \\
\hline Abrasion & 37 & -0.321 & 0.052 \\
\hline Orodental trauma & 48 & -0.311 & 0.031 \\
\hline Thirst & 12 & -0.096 & 0.766 \\
\hline Normal & 0 & - & - \\
\hline
\end{tabular}

$p=0.030)$. This was not seen for shivering, abrasions, sore throat and thirst (Table III). On comparing the preferences of younger (age $\leq 50$ years) versus older (age $>50$ years) patients (Table IV), a significant difference was seen in their ranking of orodental trauma $(p<0.05)$. However, this was not accompanied by a difference in their willingness to pay. Analysis of other subgroups reinforced the observation that significant ranking differences did not translate equally to patients' willingness to pay (Table IV). Sore throat was of greater concern for subsidised patients, those with previous anaesthesia experience and those with previous adverse post-anaesthetic outcomes $(p<0.05)$. Avoidance of nausea was ranked as more important by women and those with more than six years of education when compared to men and patients with up to six years of education, respectively $(p<0.05)$. Additionally, somnolence was of greater concern to patients with up to six years of education compared to those with over six years of education $(p<0.05)$. Besides the observed disparity between rankings and willingness to pay, the subgroup analysis identified possible subsets of patients for future targeted measures.

\section{DISCUSSION}

In our study, we found that pain, vomiting and nausea were the top three adverse post-anaesthetic outcomes of concern

Table IV. Subgroup analysis showing significant differences in ranking and relative value scores of post-anaesthesia adverse outcome.

\begin{tabular}{|c|c|c|c|c|}
\hline \multirow[t]{2}{*}{ Adverse outcome } & \multicolumn{2}{|c|}{ Median (interquartile range) } & \multicolumn{2}{|c|}{ p-value } \\
\hline & Rank & Relative value & Rank & Relative value \\
\hline Orodental trauma & & & 0.005 & 0.667 \\
\hline Age $\leq 50$ yr $(n=54)$ & $5.00(3.75-7.00)$ & $20.00(10.00-37.50)$ & & \\
\hline Age $>50$ yr $(n=78)$ & $6.00(5.00-8.00)$ & $20.00(10.00-50.00)$ & & \\
\hline \multicolumn{5}{|l|}{ Sore throat } \\
\hline Subsidised $(n=91)$ & $6.00(4.00-7.00)$ & $11.50(10.00-26.25)$ & & \\
\hline Paying class $(n=41)$ & $6.00(5.00-7.00)$ & $10.00(10.00-30.00)$ & & \\
\hline History of previous anaesthesia & & & 0.004 & 0.478 \\
\hline Previous experience $(n=95)$ & $5.00(4.00-7.00)$ & $11.00(10.00-30.00)$ & & \\
\hline No previous experience $(n=37)$ & $7.00(6.00-7.00)$ & $11.00(10.00-20.00)$ & & \\
\hline History of adverse post-anaesthetic outcome & & & 0.044 & 0.559 \\
\hline No previous adverse outcome $(n=69)$ & $6.00(5.00-7.00)$ & $12.00(10.00-32.50)$ & & \\
\hline \multicolumn{5}{|l|}{ Nausea } \\
\hline Gender & & & 0.031 & 0.207 \\
\hline Men $(n=64)$ & $3.00(2.00-5.75)$ & $20.00(10.00-23.75)$ & & \\
\hline Women $(n=68)$ & $3.00(2.00-4.00)$ & $25.00(16.50-45.00)$ & & \\
\hline Education & & & 0.032 & 0.330 \\
\hline$\leq 6 \operatorname{yr}(n=25)$ & $3.00(3.00-6.50)$ & $16.50(10.00-27.50)$ & & \\
\hline$>6$ yr $(n=97)$ & $3.00(2.00-4.00)$ & $20.00(10.00-40.00)$ & & \\
\hline Shivering & & & 0.037 & 0.406 \\
\hline Men $(n=64)$ & $6.00(5.00-8.00)$ & $10.00(10.00-16.00)$ & & \\
\hline Women $(n=68)$ & $5.00(3.25-7.00)$ & $20.00(10.00-20.00)$ & & \\
\hline$>6$ yr education $(n=97)$ & $7.00(5.00-8.50)$ & $10.00(10.00-20.00)$ & & \\
\hline
\end{tabular}


among Singapore patients, corresponding with previous Western studies. ${ }^{(9)}$ Our participants were willing to pay half of their assigned SGD 100 to prevent the occurrence of pain (median amount SGD 50). This was two-thirds more than the secondranked outcome, vomiting (median amount SGD 30). This difference in valuation was smaller in studies among Western populations, where participants were willing to pay $\$ 18.05$ and $\$ 26.10$ for their first-ranked outcome, an amount that was only $\$ 0.19$ and $\$ 9.20$ more than the second-ranked outcome in the studies of Macario et $\mathrm{al}^{(9)}$ and Jenkins et $\mathrm{al}^{\left({ }^{(10)}\right.}$ respectively. The spending difference observed between the first-ranked and subsequent outcomes was possibly associated with differences in money management between various cultures. Generally, Asians tend to value thriftiness and spend selectively on items that they attribute the greatest importance to. ${ }^{(18)}$ Patients' willingness to pay to avoid pain was also highlighted in a previous study. ${ }^{(19)}$

Correlation between the ranking and relative value of each adverse outcome was only found for pain, vomiting, nausea, orodental trauma and somnolence. However, this correlation was not seen for shivering, abrasions and sore throat, which had an equal median ranking as orodental trauma (Table II). This discrepancy was not seen in other studies, which showed clear associations between rank order and money allocation - a stepwise decrease in rank was mirrored by a stepwise decrease in monetary allocation. ${ }^{(9,10)}$ Our results may show the impact of other influences on our patients, such as the permanence of the adverse outcome and local healthcare economics. In the descriptions provided to patients, we did not specify the duration of the undesired outcomes, and orodental trauma would be viewed as being more permanent than other undesired outcomes. In addition, some participants explained that they were more willing to pay to avoid outcomes that required longer periods of recovery, required additional treatment or had a permanent effect needing repair, such as orodental trauma. Others highlighted that the disparity in the cost of treatments among the undesirable outcomes influenced their spending choices. For example, dental procedures needed for orodental trauma were likely to be more expensive, with little to no insurance coverage, when compared to medications that would be needed to treat transient nausea. In Singapore, dental procedure fees range from SGD 150-10,700. The national healthcare savings and co-insurance scheme, known as Medisave and MediShield Life, respectively, has specific exceptions for the medical conditions for which it can be used. ${ }^{(20)}$ It could be inferred that the amount of out-of-pocket payments for treatment of complications was a factor that influenced patients' willingness to avoid undesirable outcomes. ${ }^{(21-24)}$

Our result showed internal consistency with our negative control. 'Normal' was ranked as the least undesirable and no one was willing to spend any hypothetical money to avoid a normal outcome. This showed that our participants understood our instructions and were able to answer the researcher's questions appropriately.

In terms of demographics, our participants represented all the major patient groups requiring perioperative care in Singapore, reflecting similar proportions for each demographic group.
Numerous studies have suggested that demographic factors such as ethnicity, age and gender influence an individual's perception of an adverse outcome. ${ }^{(16)}$

Our study identified various subgroups that were more concerned about certain adverse outcomes: younger patients (orodental trauma); government-subsidised patients (sore throat); previous anaesthesia experience (sore throat); women (nausea); previous post-anaesthesia adverse outcomes (sore throat); and more than six years of education (nausea). However, the statistically significant differences in ranking did not translate into differences in terms of patients' willingness to pay. While we recognise that our subgroup findings may lack sufficient power, the discrepancy observed between ranking and willingness to pay could possibly be explained by the complex interaction of factors at play, including the treatment costs discussed previously and individual perceptions of permanence in outcome.

This study was not without limitations. The wide distributive variation of adverse post-anaesthetic outcomes surveyed was a potential limitation for analysis. It reflected that an element of individual choice and various multiple factors, which are not fully accounted for, contributed to the final rank choice of patients. One of the confounding factors could be that our description of undesired outcomes was brief and did not state duration, and hence was open to participants' interpretation. In recognition of this shortcoming, analysis with medians and non-parametric statistical tests were used to minimise the effect of outliers and arrive at findings that would be more reflective of our study population.

Our survey sample of 132 patients was small and resulted in underpowered subgroup analysis. Given its small sample size, our study would have more difficulty verifying small differences compared to a study with a larger cohort size. However, our sample size was comparable to previous studies conducted in this area. ${ }^{(9)}$ Finally, while this study was conducted in Singapore - a multicultural society - caution should be exercised when generalising results to the rest of Asia, where large variations in culture, developmental status and healthcare practices exist.

As our study limited patients to ten preselected adverse postanaesthetic outcomes, other outcomes, such as intraoperative awareness, may have been overlooked. Future studies could include a qualitative study to determine what outcomes would be deemed most adverse by patients and also incorporate more study variables. Translation of the questionnaire into more languages should be considered to achieve a more representative population, as well as gain higher participation numbers to power the subgroup analysis and prove causation.

Our study highlighted that patients' expectation and perception of undesirable outcomes are complex issues with no one-size-fits-all answer. In clinical practice, we often overlook patients' needs and wishes due to a lack of understanding or willingness to explore their concerns. Patient-centred care aims to provide care based on patients' needs, values and wishes so as to gain trust and result in greater satisfaction, more appropriate prescription and more efficient practice. ${ }^{(1,25)}$ Inter-participant and inter-study variations that are observed highlight the diversity 
among patients. While we seek to find common ground to optimise and bundle care for the majority of patients and identified subgroups, it is important for physicians to remember that there is no better substitute for time and effort when it comes to understanding and personalising patient care.

Several implications arise from this study. First, our results could help health service providers to refocus their resources. For example, as we found that pain, nausea and vomiting were highly undesired outcomes, clinicians and healthcare professionals should dedicate some preoperative time to patient education on pain management ${ }^{(27-30)}$ and postoperative acute pain management. A previous study ${ }^{(26)}$ has found that overall pain management systems can be improved through preoperative education on realistic pain expectations and coping strategies. Second, our study showed that our patients had varying opinions on the lower-ranking adverse outcomes; this should serve as a reminder to anaesthesiologists of the importance of patient-centred care, which is to realise the different concerns of each patient and address them accordingly. We have emphasised communication between the physician and patient to better identify the patient's concerns and expectations. Communication can not only bring about higher-quality outcomes and greater satisfaction, but also give physicians the ability to detect problems earlier and thereby prevent medical crises or the need for expensive interventions. ${ }^{(31)}$

This study examined the Singapore perspective on undesired post-anaesthesia outcomes and patients' willingness to pay to avoid these outcomes. We conclude that anaesthesiologists, as perioperative care physicians, play pivotal roles in perioperative education, communication and management of post-anaesthesia adverse outcomes. As our healthcare service becomes more patient-centred, it is critical to improve the patient experience. By identifying the patient's understanding, perception, fears and expectations, we can minimise the misalignment of expectations between physician and patients and provide better patient outcomes.

\section{REFERENCES}

1. Epstein RM, Franks P, Shields CG, et al. Patient-centered communication and diagnostic testing. Ann Fam Med 2005; 3:415-21.

2. Apfel CC, Läärä E, Koivuranta $M$, Greim CA, Roewer N. A simplified risk score for predicting postoperative nausea and vomiting: conclusions from crossvalidations between two centers. Anesthesiology 1999; 91:693-700.

3. Koivuranta $M$, Läärä $E$, Snåre $L$, Alahuhta $S$. A survey of postoperative nausea and vomiting. Anaesthesia 1997; 52:443-9.

4. Sinclair DR, Chung F, Mezei G. Can postoperative nausea and vomiting be predicted? Anesthesiology 1999; 91:109-18.

5. Apfelbaum J, Chen C, Mehta SS, Gan TJ. Postoperative pain experience: results from a national survey suggest postoperative pain continues to be undermanaged. Anesth Analg 2003; 97:534-40.
6. Gan TJ, Diemunsch P, Habib AS, et al; Society for Ambulatory Anesthesia. Consensus guidelines for the management of postoperative nausea and vomiting. Anesth Analg 2014; 118:85-113.

7. Grol R, Grimshaw J. From best evidence to best practice: effective implementation of change in patients' care. Lancet 2003; 362:1225-30.

8. Gan TJ, Meyer TA, Apfel C, et al; Society for Ambulatory Anesthesia. Society for Ambulatory Anesthesia guidelines for the management of postoperative nausea and vomiting. Anesth Analg 2007; 105:1615-28.

9. Macario A, Weinger M, Carney S, Kim A. Which clinical anesthesia outcomes are important to avoid? The perspective of patients. Anesth Analga 1999; 89:652-8.

10. Jenkins K, Grady D, Wong J, et al. Post-operative recovery: day surgery patients' preferences. Br J Anaesth 2001; 86:272-4.

11. Ayanian JZ, Zaslavsky AM, Guadagnoli E, et al. Patients' perceptions of quality of care for colorectal cancer by race, ethnicity, and language. J Clin Oncol 2005; 23:6576-86.

12. McLaughlin LA, Braun KL. Asian and Pacific Islander cultural values: considerations for health care decision making. Health Soc Work 1998; 23:116-26.

13. Gillam $\mathrm{S}$, Jarman $\mathrm{B}$, White $\mathrm{P}$, Law R. Ethnic differences in consultation rates in urban general practice. BMJ 1989; 299:953-7.

14. Taira D, Safran DG, Seto TB, et al. Do patient assessments of primary care differ by patient ethnicity? Health Serv Res 2001; 36(6 Pt 1):1059-71.

15. Haviland MG, Morales LS, Reise SP, Hays RD. Do health care ratings differ by race or ethnicity? Jt Comm J Qual Saf 2003; 29:134-45.

16. Yek JL, Lee AK, Tan JA, et al. Defining reasonable patient standard and preference for shared decision making among patients undergoing anaesthesia in Singapore. BMC Med Ethics 2017; 18:6.

17. Lau HP, White MP, Schnall S. Quantifying the value of emotions using a willingness to pay approach. Happiness Stud 2013; 14:1543-61.

18. Khan H. Social Policy in Singapore: a Confucian Model? Available at: http:// documents.worldbank.org/curated/en/193101468758956946/016824232_20 02113371001150/additional/multiopage.pdf. Accessed April 12, 2018.

19. van den Bosch J, Bonsel GJ, Moons KG, Kalkman CJ. Effect of postoperative experiences on willingness to pay to avoid postoperative pain, nausea, and vomiting. Anesthesiology 2006; 104:1033-9.

20. Ministry of Health, Singapore. Dental Fees. Available at: https://www.moh. gov.sg/content/moh_web/home/costs_and_financing/Dental_Fee_Info.html. Accessed April 12, 2018.

21. O'Donnell O, van Doorslaer E, Rannan-Eliya RP, et al. Who pays for health care in Asia? J Health Econ 2008; 27:460-75.

22. van Doorslaer E, O'Donnell O, Rannan-Eliya RP, et al. Effect of payments for health care on poverty estimates in 11 countries in Asia: an analysis of household survey data. Lancet 2006; 368:1357-64.

23. McIntyre D, Thiede M, Dahlgren G, Whitehead M. What are the economic consequences for households of illness and of paying for health care in low- and middle-income country contexts? Soc Sci Med 2006; 62:858-65.

24. Lu JF, Hsiao WC. Does universal health insurance make health care unaffordable? Lessons from Taiwan. Health Aff (Millwood) 2003; 22:77-88.

25. Fiscella K, Meldrum S, Franks $P$, et al. Patient trust: is it related to patient-centered behavior of primary care physicians? Med Care 2004; 42:1049-55.

26. Svensson I, Sjöström B, Haljamäe H. Influence of expectations and actual pain experiences on satisfaction with postoperative pain management. Eur J Pain $2001 ; 5: 125-33$.

27. Harsoor S. Emerging concepts in post-operative pain management. Indian J Anaesth 2011; 55:101-3.

28. Jackson T, Pope L, Nagasaka T, et al. The impact of threatening information about pain on coping and pain tolerance. Br J Health Psychol 2005; 10(Pt 3):441-51.

29. Mossey J. Defining racial and ethnic disparities in pain management. Clin Orthop Relat Res 2011; 469:1859-70.

30. Wandner LD, Scipio CD, Hirsh AT, Torres CA, Robinson ME. The perception of pain in others: how gender, race, and age influence pain expectations. J Pain 2012; 13:220-7.

31. Ha JF, Longnecker N. Doctor-patient communication: a review. Ochsner J 2010; 10:38-43. 